\title{
Magnetic Losses Evolution of Ferritic Fe-Si Steel Subjected to Temper Rolling at Elevated Temperature
}

\begin{abstract}
I. Petryshynets*, F. Kováč, L. Falat, V. Puchý and M. Šebek
Institute of Materials Research, Slovak Academy of Sciences, Watsonova 47, 04001 Košice, Slovakia

The effects of heat transport phenomena and deformation gradient through the sheet cross-section on the microstructure, texture and magnetic properties of non-oriented $\mathrm{Fe}-\mathrm{Si}$ steel were investigated. In order to achieve desired conditions for the steel microstructural and textural parameters improvement, a temper rolling process at elevated temperature was examined in combination with subsequent short term annealing, employing fast heating rate in laboratory conditions. The experimental material was represented by conventional medium silicon, vacuum degassed non-oriented steel that was taken directly from industrial line after final annealing. Performed electron back scattered diffraction measurements revealed that the experimental steel, treated by our proposed thermomechanical treatment, was characterized by coarse-grained microstructure with enhanced intensity of rotating cube texture. The magnetic losses of experimental samples were measured in ac magnetic field with $50 \mathrm{~Hz}$ frequency on the toroid with external and internal diameters of $25 \mathrm{~mm}$ and $15 \mathrm{~mm}$, respectively. These measurements with magnetic field intensity of $2500 \mathrm{~A} / \mathrm{m}$ showed that the application of our suggested treatment led to clear power losses reduction of the investigated material from initial $9.9 \mathrm{~W} / \mathrm{kg}$ to $6.4 \mathrm{~W} / \mathrm{kg}$, i.e. by more than $35 \%$.
\end{abstract}

DOI: 10.12693/APhysPolA.133.1065

PACS/topics: 61.72.-y, 75.50.Vv, 81.40.Ef, 61.72.Cc

\section{Introduction}

Non-oriented (NO) Fe-Si steels belong to important group of the soft magnetic materials that are typically used as core parts in a variety of electrical rotating equipment such as electrical motors, power generators, alternators, etc. [1]. That is why the non-oriented steels have to possess appropriate magnetic properties in all plane directions of steels. The magnetic properties of finally fabricated NO silicon steels are strongly affected by chemical composition, grains size of microstructure, residual stresses, and occurring crystallographic texture components [2]. The existing magnetic texture in polycrystalline materials determines the magnetization behavior at higher values of applied external magnetic field. The microstructural parameters affect mainly the magnetizing behavior at low and medium values of the applied external magnetic field and the hysteresis losses. An optimum grain size after final annealing is preferable for low values of the specific magnetic losses. A low intensity of the $\gamma$-fiber texture $\{111\}\langle u v w\rangle$ and $\alpha$-fiber texture $\{h k l\}\langle 011\rangle$ as well as a high intensity of the magnetic relevant texture components and fibers, like the Goss $\{011\}\langle 100\rangle$, cube $\{100\}\langle 001\rangle$, rotated cube $\{100\}\langle 011\rangle$ components and $\eta$-fibers $\langle 001\rangle / \mathrm{RD}$ are generally desirable for an excellent magnetization behavior of NO electrical steels $[3,4]$.

The magnetic properties of electrical steels such as magnetization curves, permeability and specific losses are, to a large extent, correlated with the microstructure

*corresponding author; e-mail: ipetryshynets@saske.sk and crystallographic texture. The resulting microstructural characteristics of materials are conditioned by the thermomechanical processing which involves a slab reheating, hot rolling, cold rolling and final recrystallization annealing [5].

Nevertheless, improvement of the electromagnetic properties can be achieved by the application of the additional technological steps, which can provide coarsegrained microstructure with desirable crystallographic orientation of the material. The industrial production of vacuum-degassed electrical steels with $\mathrm{C} \approx 0.003 \%$ leads to new theories of microstructure development in these kinds of steels.

Our previous works $[6,7]$ were focused on optimizing microstructural and textural parameters of $\mathrm{Fe}-\mathrm{Si}$ electrical steel sheets with low and high Si contents by means of columnar grains growth. The present work considers a method of coarse-grained microstructure development in $\mathrm{NO}$ vacuum-degassed steels with medium silicon content. The strain-induced grain boundary motion mechanism (SIMB) with combination of temperature gradient through the cross-section of plane was applied to the investigated steel to achieve the required large grained microstructure with a high intensity of rotating cube $\{100\}\langle u v w\rangle$ and/or the Goss crystallographic orientation. This proposed mechanism provides grain growth from both surfaces to the mid-thickness of the steel sheet. In order to achieve the high intensity of microstructure evolution the SIBM was invoked by small cold rolling deformation (temper rolling) at elevated temperature.

\section{Experimental procedure}

The used experimental material was a vacuum degassed NO steel with following chemical composition in 
wt $\%: C=0.003, \mathrm{Si}=1.25, \mathrm{Mn}=0.183, \mathrm{P}=0.039$, $\mathrm{Al}=0.28$. A strip of $0.5 \mathrm{~mm}$ in thickness was taken from industrial line after final continuous annealing and marked as SF samples.

Then this steel material was variously treated under laboratory conditions. Experimental samples were heated up to $250^{\circ} \mathrm{C}$ and thereupon (during $c a .2 \mathrm{~s}$ ) were temper rolled within one pass at particular rolling reductions. The values of experimental temper rolling reduction were in the range from 2 to $10 \%$.

A dynamic heat treatment was applied to temper rolled strips to study grain growth phenomena in the material. The samples with planar dimensions of $30 \mathrm{~mm} \times 10 \mathrm{~mm}$ were heated up to different temperatures in the range $850-950{ }^{\circ} \mathrm{C}$ at a heating rate of more than $25^{\circ} \mathrm{C} / \mathrm{s}$ and were kept at the chosen temperature for $5 \mathrm{~min}$. The heat treatment was carried out in a dry hydrogen atmosphere.

The watt losses of the experimental samples were measured in AC magnetic field with $50 \mathrm{~Hz}$ frequency on the toroid with external and internal diameters of $25 \mathrm{~mm}$ and $15 \mathrm{~mm}$, respectively. These measurements were performed by using commercial REMACOMP C-705 system from MAGNET-PHYSIK Dr. Steingroever GmbH.

The nanohardness testing was carried out using Nanoindenter Agilent G200. These measurements were performed on the surface of longitudinal cross-sections of the experimental samples.

The most representative samples were chosen for the microstructure and texture analysis. The texture analysis was carried out by means of electron back scattered diffraction (EBSD) method in the normal direction plane for each sample of $25 \mathrm{~mm} \times 10 \mathrm{~mm}$ in size. The JEOL JSM 7000F FEG scanning electron microscope was used to perform the texture analysis. The EBSD patterns were detected by the "Nordlys-I" EBSD detector. The data obtained were processed by the CHANNEL-5, HKL software package.

\section{Results and discussion}

The initial microstructure of the investigated steel in as-received state obtained from industrial line after final annealing (SF sample) is presented in Fig. 1a. As one can see, the sample finished in industrial condition is characterized by a quite fine-grained and homogeneous microstructure with mean grain size of $25-35 \mu \mathrm{m}$. The microstructure evolution of recrystallized SF samples after temper rolling at elevated temperature with subsequent annealing in dynamic conditions is presented in Fig. 1be. These images clearly demonstrate the effects of individual thermomechanical treatments on the motion of grain boundaries in the investigated steel, depending on annealing temperature and applied deformation.

The microstructure of SF sample after annealing at $850^{\circ} \mathrm{C}$ for $5 \mathrm{~min}$ in hydrogen atmosphere is shown in Fig. 1b. Here, the mentioned sample was subjected to the temper rolling process with $2 \%$ of deformation. This microstructure is characterized by a commencement of the
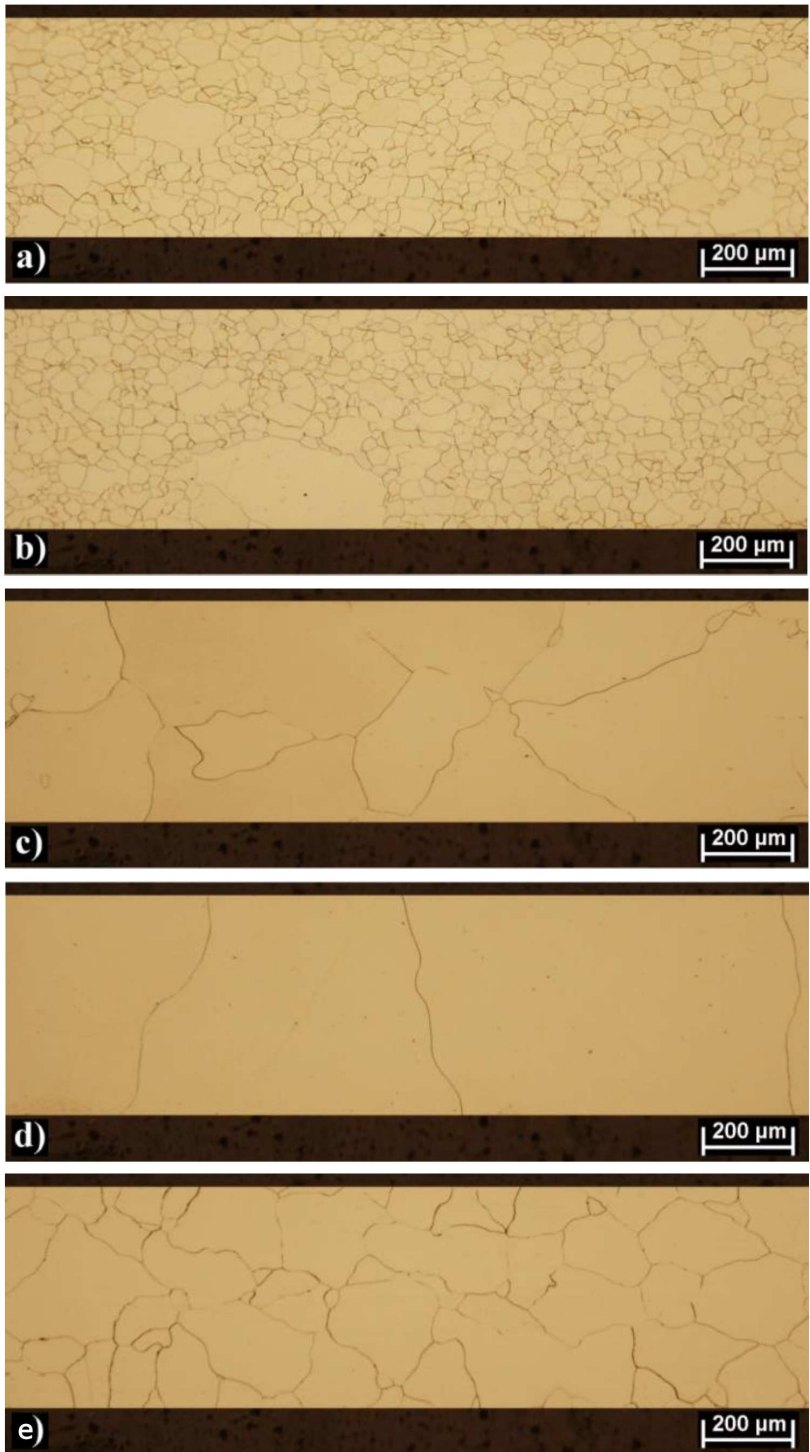

Fig. 1. The microstructures of SF steel: (a) sample after final annealing in industrial conditions, temper rolled samples with (b) $2 \%$ of deformation annealed at $850{ }^{\circ} \mathrm{C}$, (c) $6 \%$ of deformation annealed at $950^{\circ} \mathrm{C}$, (d) $4 \%$ of deformation annealed at $950^{\circ} \mathrm{C}$, (e) $8 \%$ of deformation annealed at $950^{\circ} \mathrm{C}$.

formation of coarse grain consuming the primary recrystallized grain matrix. It is well seen that this grain grows towards the center of the sample. The average grain size of regarded microstructure is about $40 \mu \mathrm{m}$. Figure 1c demonstrates the grain growth evolution of investigated sample with $6 \%$ of deformation after annealing at $950^{\circ} \mathrm{C}$. As one can see, the microstructure is characterized by relatively homogeneous grain matrix with average grain size of $350 \mu \mathrm{m}$. The microstructure of experimental steel after temper rolling with $4 \%$ thickness reduction and following annealing at $950{ }^{\circ} \mathrm{C} / 5$ min exhibits completely coarsened grains, see Fig. 1d. In this case, the experimental steel shows complete coarse grain growth with the maximal average grain size of $560 \mu \mathrm{m}$. 
The microstructure of experimental steel after temper rolling with $8 \%$ of deformation and annealed $950{ }^{\circ} \mathrm{C}$ is presented in Fig. 1e. As one can see, the microstructure of this sample is characterized by homogeneous distribution of grains with average size of $220 \mu \mathrm{m}$.

Dependences describing relations between average grain sizes and value of annealing temperatures at fixed values of applied deformation are presented in Fig. 2a. The each deformed samples was annealed at five different temperatures during $300 \mathrm{~s}$. It is well seen that such kind of dependence gives unambiguous information about grain size and describes dynamics of microstructure changes. Comparison of the mentioned dependences and microstructure of the investigated materials between each other leads to suggestion that dynamics of grain growth strongly depends on the magnitude of applied deformation. It is necessary to note that the most pronounced changes in materials microstructure are in range of low deformations between $2 \%$ and $6 \%$. The subsequent annealing in the range of $900^{\circ} \mathrm{C}-950{ }^{\circ} \mathrm{C}$ applied for the investigated samples leads to dynamic development of the coarse-grained microstructure. On the other hand, low dynamics of grain growth was observed in the samples deformed in the range of reductions lying between $8 \%$ and $10 \%$, see Fig. 2a. Moreover, the grains sizes and microstructure characteristics are not significantly influenced by annealing temperatures and times as it was observed for lower deformations $(\varepsilon \approx 2-4 \%)$. The presented dependences of microstructure development in the investigated material leads to suggestion that the applied deformation during temper rolling has an important impact not only on the final grain size and grain growth rate but also on the mechanism of grain growth process.
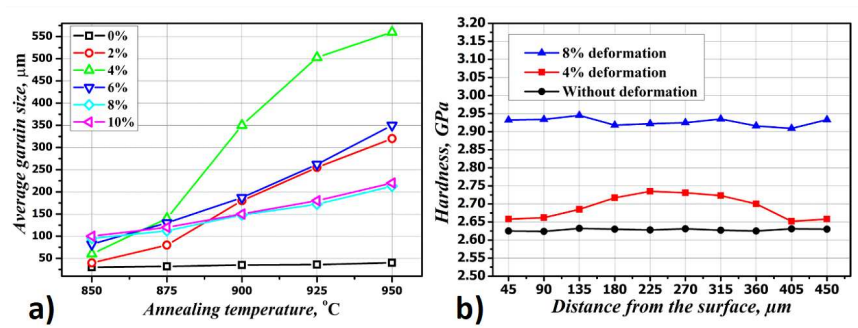

Fig. 2. Dependence of (a) average grain size on applied deformation in SF steel annealed at different temperatures after temper rolling process, (b) nano hardness on cross - section of experimental steels.

To investigate the accumulation of deformation energy and its dependence on the distance of the surface in small volumes in temper rolling samples at elevated temperature, the nanohardness test was carried out within the present work. Hence, this method is characterized by high measurement accuracy. It is well known that steel sheets after the deformation possess accumulated deformation energy that could be detected through the hardness measurements [8]. On the basis of this assumption, the nanohardness measurements across the thickness were done on SF samples without deformation (black line) and samples taken after temper rolling with $4 \%$ (red line) and $8 \%$ (blue line) reduction, see Fig. 2b. Here, the black curve, which was obtained for the sample without temper rolling, describes the uniform distribution of nanohardness values about $2.625 \mathrm{GPa}$ through the whole steel thickness. The similar linear profile of nanohardness was obtained for temper rolled sample with $8 \%$ of deformation. The average value of nanohardness for this dependence is $2.925 \mathrm{GPa}$, see blue line in Fig. 2b. In the case of the samples after temper rolling with $4 \%$ deformation, the corresponding dependence describes the deformation gradient from the surface of sample to its central part (see red line in Fig. 2b). The obtained results indicated that the application of optimal deformation conditions (with sample preheating before rolling reduction) leads to a generation of deformation gradient through the cross section from the surface to the central region. This is due to existing anisotropy of temperature field through the thickness of the steel sheet when the center of the material has a higher temperature than the surface region.

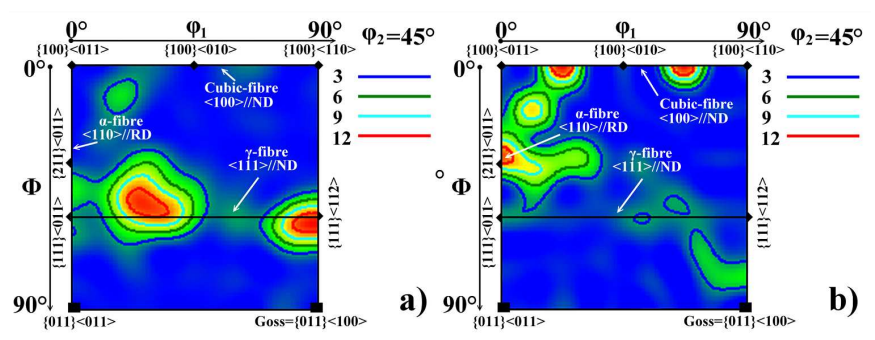

Fig. 3. The ODF section at $\varphi_{2}=45^{\circ}$ represents the evolution of orientation density along $\alpha-, \gamma$ - and cubicfibre for SF samples taken: (a) from industrial line and (b) after temper rolling with $4 \%$ of deformation and subsequent laboratory annealing at $950^{\circ} \mathrm{C} / 5 \mathrm{~min}$.

Figure 3 shows the results obtained from EBSD measurements which present intensities of particular crystallographic texture components. Here, the EBSD analysis in the form of orientation distribution function (ODF) taken at $\varphi_{2}=45^{\circ}$ was carried out on the SF samples presented in Fig. 1a and d, see Fig. 3a and b, respectively. The common texture can be represented also by orientation fibers in the Euler space taken from ODF. The most relevant fibers for the investigated non-oriented steel are $\gamma-, \alpha-$, and the Cube-fiber. As one can see, Fig. 3a shows that SF sample taken from industrial line is characterized by dominance of $\gamma$ - and $\alpha$-fiber. It is well know that the $\gamma$-fiber represents the intensity of deformed texture component $\{111\}\langle 011\rangle$ which is undesirable for non-oriented silicon steels. On the other hand, the EBSD analysis shows very strong intensity of the Cube-fiber of coarsegrained microstructure which was achieved after temper rolling with $4 \%$ of deformation, see Figs. $3 \mathrm{~b}$ and 1 d. This fact suggests that huge grains with rotating cube crystallographic orientation $\{100\}\langle u v w\rangle$ were achieved in the specimens that were treated by our proposed thermomechanical method using the combination of temper rolling 
process with small reduction and subsequent final annealing in dynamic conditions.

Precise estimation of the relationship between microstructure, texture and domain wall motion in the experimental NO steel can be obtained through measurements of magnetic watt losses. Table I shows the dependence of measured AC magnetic losses on the applied deformation for selected samples of the investigated material. The watt losses of the experimental samples were measured in $\mathrm{AC}$ magnetic field with $50 \mathrm{~Hz}$ frequency and different magnetic field parameters on the toroid with external diameter of $25 \mathrm{~mm}$ and internal diameter of $15 \mathrm{~mm}$. The magnetic loss of the sample taken after the industrial final continuous annealing was also measured. Here, the performed magnetic measurements are representative and made in order to distinguish specimens after different steps of thermomechanical treatment. Additionally, the measurements clearly show the differences between magnetic properties of the specimens treated under laboratory and industrial conditions. The lowest value of the magnetic loss for the experimental steel annealed in laboratory conditions was $6.4 \mathrm{~W} / \mathrm{kg}$. This result represents a significant decrease of the watt loss, compared to the as-received state showing the watt loss of $9.9 \mathrm{~W} / \mathrm{kg}$.

The magnetic properties of chosen samples of investigated steel.

TABLE I

\begin{tabular}{|c|c|c|c|c|c|c|c|}
\hline \multirow{3}{*}{$\begin{array}{l}\text { Characteristic } \\
\text { of magnetic field }\end{array}$} & \multirow{3}{*}{$f,[\mathrm{~Hz}]$} & \multirow{2}{*}{\multicolumn{2}{|c|}{$\begin{array}{l}\text { SF steel taken after final } \\
\text { industrial annealing }\end{array}$}} & \multicolumn{4}{|c|}{ SF steel treated at laboratory conditions } \\
\hline & & & & \multicolumn{2}{|c|}{$\varepsilon=6 \%, T=950^{\circ} \mathrm{C}, t=5 \mathrm{~min}}$. & \multicolumn{2}{|c|}{$\varepsilon=4 \%, T=950^{\circ} \mathrm{C}, t=5 \mathrm{~min}$} \\
\hline & & {$[\mathrm{A} / \mathrm{m}]$} & {$[\mathrm{W} / \mathrm{kg}]$} & {$[\mathrm{A} / \mathrm{m}]$} & {$[\mathrm{W} / \mathrm{kg}]$} & {$[\mathrm{A} / \mathrm{m}]$} & {$[\mathrm{W} / \mathrm{kg}]$} \\
\hline$B_{\max }=1.5 \mathrm{~T}$ & \multirow{4}{*}{50} & 216 & 9.7 & 152 & 8.9 & 134 & 7.8 \\
\hline$H_{\max }=2500 \mathrm{~A} / \mathrm{m}$ & & 218 & 9.9 & 118 & 6.9 & 112 & 6.4 \\
\hline$H_{\max }=5000 \mathrm{~A} / \mathrm{m}$ & & 280 & 14.0 & 159 & 9.8 & 158 & 9.4 \\
\hline$H_{\max }=10000 \mathrm{~A} / \mathrm{m}$ & & 347 & 18.4 & 216 & 14.3 & 212 & 13.4 \\
\hline
\end{tabular}

The elaborated deformation state provides condition for the deformation induced ferrite grain growth that is directed from the surface to the central region of the sheet. Moreover, such deformation state leads to selectivity in the growth of grains with favorable rotating cub $\{100\}\langle 0 v w\rangle$ and the Goss $\{110\}\langle 001\rangle$ texture components. Such characteristics of microstructure and texture have a positive impact on soft magnetic properties including a decrease of coercitivity, which in turn favorably affects the watt losses.

\section{Conclusions}

In summary, we have shown by means of kinetic analysis of microstructural development that the complex processes caused by rapid heating in combination with strain-induced grain boundary motion mechanism allow achieve coarse grain growth in fully processed nonoriented electrical steel. A strong temperature and optimal deformation gradients parallel to the sheet normal lead to abnormal grain boundary migration toward the mid-thickness part of the steel.

The main factors controlling coarse-grained microstructure evolution during our proposed thermomechanical treatment are: temperature of annealing, heating rate and value of applied deformation induced by temper rolling process at elevated temperatures.

\section{Acknowledgments}

This work was carried out within the project, which is supported by the Slovak Research and Development Agency under the contract No. APVV-15-0259. This work was also partially supported by the Slovak Grant Agency VEGA, project No. 2/0081/16 and
No. 2/0066/18. Also, the work was realized within the frame of the project ITMS 26220220061 and ITMS 26220220064 .

\section{References}

[1] D. Steiner Petrović, D. Mandrino, Mater. Charact. 62, 503 (2011).

[2] J.B. Salinas, A.R. Salinas, J. Iron Steel Res. Int. 23, 166 (2016).

[3] J. Schneider, G. Li, A. Franke, B. Zhou, J. Magn. Magn. Mater. 424, 26 (2017).

[4] M. Mehdi, Y. He, E.J. Hilinski, A. Edrisy, J. Magn. Magn. Mater. 429, 148 (2017).

[5] J.J. Sidor, K. Verbeken, E. Gomes, J. Scneider, P.R. Calvillo, L.A.I. Kestens, Mater. Charact. 71, 49 (2017).

[6] I. Petryshynets, F. Kovac, J. Marcin, I. Škorvanek, Acta Phys. Pol. A 126, 182 (2014).

[7] F. Kováč, I. Petryshynets, V. Puchy, M. Šebek, J. Maruš, J. Electr. Eng. 66, 86 (2015).

[8] F.J. Humphreys, M. Hatherly, Recrystallization and Related Annealing Phenomena, BPC Wheatons, 1995, p. 498. 